\title{
Model-independent test of the truncated crater function theory of surface morphology evolution during ion bombardment
}

\author{
Joy C. Perkinson, ${ }^{1, *}$ Eitan Anzenberg, ${ }^{2, *}$ Michael J. Aziz, ${ }^{1}$ and Karl F. Ludwig, Jr. ${ }^{2}$ \\ ${ }^{1}$ Harvard School of Engineering and Applied Sciences, Cambridge, Massachusetts 02138, USA \\ ${ }^{2}$ Department of Physics, Boston University, Boston, Massachusetts 02215, USA \\ (Received 15 February 2013; revised manuscript received 4 March 2014; published 26 March 2014)
}

\begin{abstract}
A broad class of "local response" theories seeks to predict morphology evolution during energetic particle irradiation in terms of average surface height response to individual impacts-an approach that has been generalized by the crater function formalism of Norris et al. [J. Phys.: Condens. Matter 21, 224017 (2009); Nat. Commun. 2, 276 (2011)]. Keeping only the terms in the crater function formalism associated with the response of a flat surface has facilitated the use of molecular dynamics simulations of individual ion impacts to predict the stability or instability of a flat surface to ion bombardment. Here we report a sensitive experimental test of this truncated crater function theory that is independent of any a priori knowledge of the crater function itself. Existing measurements for $1 \mathrm{keV} \mathrm{Ar}+/ \mathrm{Si}$ and $\mathrm{Kr}^{+} / \mathrm{Ge}$ are inconsistent with the predictions of truncated crater function theory, for any conceivable crater function, at high bombardment angles. The failure of the theory suggests that the prediction of surface evolution from simulations of single-ion impacts will be more challenging than had been assumed.
\end{abstract}

DOI: 10.1103/PhysRevB.89.115433

PACS number(s): 81.16.Rf, 68.49.Sf, 81.65.Cf

Energetic particle irradiation of solid surfaces can produce a variety of nanoscale surface topographies, including dots, ripples, holes, and ultrasmoothing [1]. The physical mechanisms governing this pattern formation are not yet well understood. The most widely investigated theoretical approaches to the phenomenon treat large-scale, long-time morphology evolution during bombardment as being due to the superposition of surface height changes associated with individual ion impacts. For simplicity, we call this broad class of theories "local response" theories. Seminal examples of local response theories are the Bradley-Harper (BH) [2] theory examining effects of erosion and the CarterVishnyakov (CV) [3] examination of "mass redistribution" (i.e., displacement of nearby atoms that are not eroded away). In their analyses the $\mathrm{BH}$ and $\mathrm{CV}$ theories used simple models for sputter erosion and atomic redistribution, respectively. More recently, Davidovitch et al. [4] showed that the surface morphology evolution can be sensitive to the details of the average local response to individual ion impacts rather than just its broad characteristics. Motivated by these results, Norris et al. [5,6] developed a general local response formalism predicting the surface evolution during ion bombardment from the moments of the crater function $\Delta h(x, y ; \theta)$ - the average change in height of a flat surface at point $(x, y)$ due to an ion impact at the origin. Here $\theta$ is the angle of ion incidence with respect to the surface normal. The crater function approach incorporates both erosive and redistributive effects, and reproduces the earlier $\mathrm{BH}$ and $\mathrm{CV}$ approaches when appropriate simple local responses are assumed [5]. A key practical goal of local response theories is to predict surface evolution during ion bombardment from knowledge of the average response to an individual impact; this can itself, in principle, be obtained from molecular dynamics (MD) simulations [6,7]. To facilitate

*J.C.P. and E.A. contributed equally to this work. use of MD simulations in predicting surface behavior, a recent simplification of the general crater function formalism, which we term "truncated" crater function theory, assumes that knowledge of the crater function formed on a flat surface is sufficient to determine the surface stability/instability in the linear stability limit of infinitesimal perturbations from a smooth, flat surface $[6,8]$. Consequently, it expresses the curvature coefficients governing surface evolution in terms of only the angular dependence of the first moment of the crater function $M^{(1)}(\theta)$.

For the case of $\mathrm{Ar}^{+}$bombardment of $\mathrm{Si}\left(\mathrm{Ar}^{+} / \mathrm{Si}\right)$, Norris et al. [6] used crater function moments calculated from MD simulations to correctly predict the $\theta \cong 45^{\circ}$ transition from stability to instability with increasing bombardment angle. In this case, they found that redistribution plays a much larger role in driving surface structure than does erosion, except possibly very near grazing incidence. This is consistent with recent in situ grazing incidence small-angle $\mathrm{x}$-ray scattering (GISAXS) measurements of the curvature coefficients in this system $[9,10]$.

A major challenge in evaluating the accuracy of the crater function approach is that experimental measurements of the crater function are unavailable and MD calculations of the function or its moments are computer-intensive and of uncertain accuracy. If a given crater function prediction made using input from MD is shown to be wrong, is the problem with the MD results or with the formalism itself? Here we point out that, independent of the shape of the crater function for a given system, the structure of the truncated form of Ref. [6] places strong constraints on the behavior of the curvature coefficients. Thus, measurements of the curvature coefficients as functions of ion incidence angle can provide sensitive tests of truncated crater function theory without a priori knowledge of the crater function or its moments.

Within linear theory, which describes surface morphology evolution at early times and low fluence, the evolution of surface height $h(x, y, t)$ during ion bombardment can be 
written as [2]

$$
\frac{\partial h(x, y, t)}{\partial t}=\left(S_{X}(\theta) \frac{\partial^{2} h}{\partial x^{2}}+S_{Y}(\theta) \frac{\partial^{2} h}{\partial y^{2}}\right)-B \nabla^{4} h,
$$

where $S_{X, Y}(\theta)$ are the curvature coefficients governing the long-wavelength (i.e., lengths on which a continuum description is accurate) behavior of the surface in the $x$ direction (parallel to the projected direction of the ion beam, or "parallel mode") and $y$ direction (perpendicular to the projected direction, or "perpendicular mode"). The coefficient $B$ represents the magnitude of ion-enhanced surface-confined viscous flow, which is always a smoothing effect. In writing Eq. (1) we neglect for simplicity the average erosion rate and slope-dependent erosion rate, which do not affect the linear stability or instability of the surface: the erosion rate can be neglected by working in the frame of reference of the average surface height, and Fourier analysis following the methods of Ref. [9] reveals that the slope-dependent erosion rate contributes only an imaginary component (traveling waves) to the perturbation's amplification rate. When either $S_{X, Y}(\theta)>0$, the surface is stable to long-wavelength fluctuations in that direction; conversely, when either $S_{X, Y}(\theta)<0$ the surface is unstable to long-wavelength fluctuations in that direction.

The truncated crater function approach of Ref. [6] expands the local response in moments of the crater function; the first moment is simply $M^{(1)}(\theta)=\int x \Delta h(x, y ; \theta) d x d y$. The theory predicts the angle-dependent curvature coefficients to be [6]

$$
\begin{aligned}
S_{X}(\theta) & =I_{0} \frac{d}{d \theta}\left[M^{(1)}(\theta) \cos (\theta)\right], \\
S_{Y}(\theta) & =I_{0} M^{(1)}(\theta) \cos (\theta) \cot (\theta),
\end{aligned}
$$

where $I_{o}$ is the ion-beam flux.

We now examine constraining predictions introduced by the theoretical approach itself. By symmetry $M^{(1)}\left(\theta=0^{\circ}\right)=$ 0 . Because we expect $M^{(1)}(\theta)$ to remain finite as $\theta \rightarrow 90^{\circ}$, the expression inside the derivative of Eq. (2) goes to zero at both $\theta=0^{\circ}$ and $\theta=90^{\circ}$. Therefore

$$
\int_{0^{\circ}}^{90^{\circ}} S_{X}(\theta) d \theta=I_{0}\left[M^{(1)}(\theta) \cos \theta\right]_{0^{\circ}}^{90^{\circ}}=0 .
$$

This is a strong constraint that is independent of the shape of the crater function itself. It states that no matter what the relative magnitudes of the contributions from erosive and redistributive effects are, there must be a properly weighted balance between regions of bombardment angles causing surface stability and instability.

A second constraint arises because both $S_{X}(\theta)$ and $S_{Y}(\theta)$ are functions of $M^{(1)}$. Therefore Eqs. (2) and (3) can be used to write $S_{X}(\theta)$ and $S_{Y}(\theta)$ in terms of each other:

$$
\begin{gathered}
S_{Y}(\theta)=\cot \theta \int_{0}^{\theta} S_{x}(q) d q ; \\
S_{X}(\theta)=\frac{d}{d \theta}\left(S_{y} \tan \theta\right) .
\end{gathered}
$$

Independent measurements of the two curvature coefficients as functions of ion incidence angle can then be compared to this self-consistency requirement of the crater function theory.

To test the truncated crater function theory, we rely on recently published sets of $S_{X}(\theta)$ and $S_{Y}(\theta)$ values for $1-\mathrm{keV}$ bombardment of $\mathrm{Ar}^{+} / \mathrm{Si}[9,10]$ and $\mathrm{Kr}^{+} / \mathrm{Ge}$ [11] using in situ GISAXS. Figures 1 and 2 reproduce the key experimental results for the angular dependence of the curvature coefficients. It is immediately clear that $\int_{x}^{S}(\theta) d \theta$ is not zero for either $\mathrm{Ar}^{+} / \mathrm{Si}$ or $\mathrm{Kr}^{+} / \mathrm{Ge}$, in contrast to the prediction of the crater function theory. For $\mathrm{Ar}^{+} / \mathrm{Si}$, the region of positive $S_{X}(\theta)$ is more than 3 times larger than the region of negative $S_{X}(\theta)$. For $\mathrm{Kr}^{+} / \mathrm{Ge}$, which has a higher critical angle for the stability-instability transition, the asymmetry is even larger. Thus, in both cases there is a surfeit of stability as compared to instability.

We can obtain more information about where problems may lie by examining the predicted relationships of Eqs. (5) and (6). To do so, the experimental $S_{X . Y}(\theta)$ results must be parameterized. In Refs. [9] and [10] it was found that for $\mathrm{Ar}^{+} / \mathrm{Si}$ the $S_{X . Y}(\theta)$ values could be fit well by a CV mass redistribution form: $S_{X}(\theta) \sim \cos 2 \theta ; S_{Y}(\theta) \sim \cos \theta$, modified with an empirical Yamamura correction factor $\exp [-\Sigma([1 / \cos (\theta)]-1)][12]$. The $\mathrm{Kr}^{+} / \mathrm{Ge}$ results [11] are qualitatively similar to the $\mathrm{Ar}^{+} / \mathrm{Si}$ results but with the transition angle from positive to negative $S_{X}(\theta)$ (i.e., from

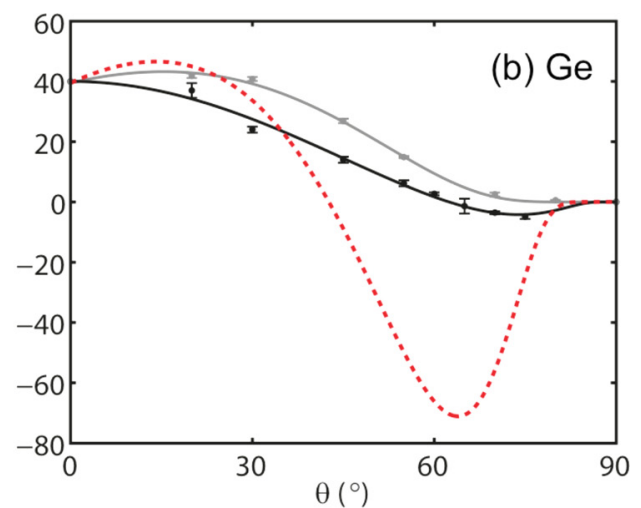

FIG. 1. (Color online) Data points: Experimental values of $S_{X}(\theta)$ (black circles) and $S_{Y}(\theta)$ (gray circles) as reported in (a) Refs. [9] and [10] for $\mathrm{Ar}^{+} / \mathrm{Si}$ and (b) Ref. [11] for $\mathrm{Kr}^{+} / \mathrm{Ge}$. The black and gray lines are fits of the experimental $S_{X}(\theta)$ and $S_{Y}(\theta)$ to the heuristic functional forms of Eqs. (7) and (8). The red dashed lines are predictions of $S_{X}(\theta)$ from $S_{Y}(\theta)$ using Eq. (6). Positive values are stabilizing and negative values are destabilizing. 

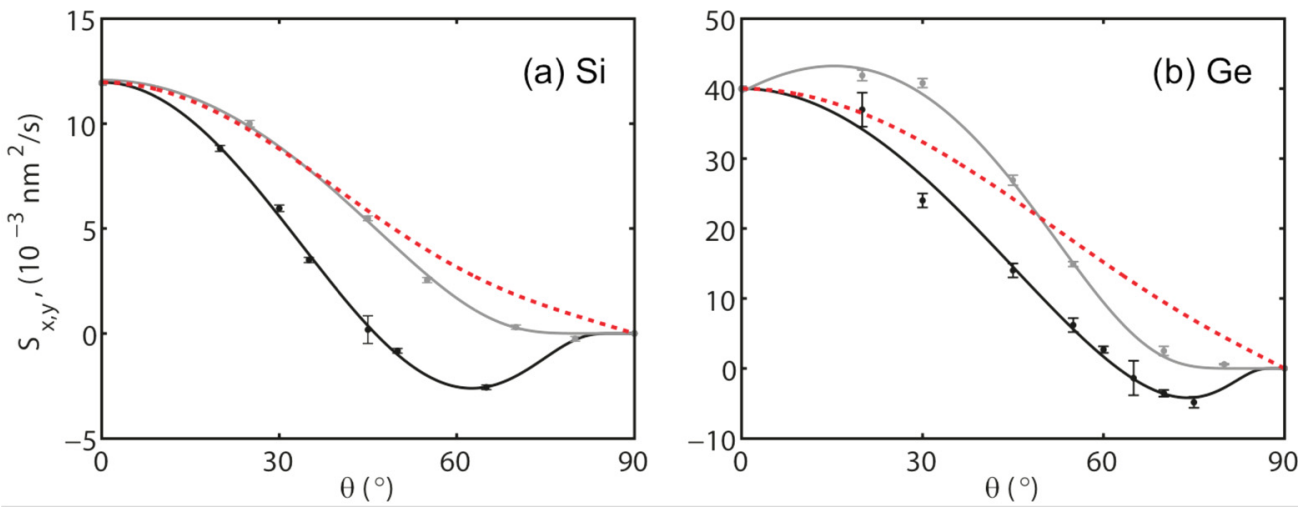

FIG. 2. (Color online) Data points: Experimental values of $S_{X}(\theta)$ (black circles) and $S_{Y}(\theta)$ (gray circles) as reported in (a) Refs. [9] and [10] for $\mathrm{Ar}^{+} / \mathrm{Si}$ and (b) Ref. [11] for $\mathrm{Kr}^{+} / \mathrm{Ge}$. The black and gray lines are fits of the experimental $S_{X}(\theta)$ and $S_{Y}(\theta)$ to the heuristic functional forms of Eqs. (7) and (8). The red dashed lines are predictions of $S_{Y}(\theta)$ from $S_{X}(\theta)$ using Eq. (5). Positive values are stabilizing and negative values are destabilizing.

stability to instability) occurring at approximately $60^{\circ}$ rather than $45^{\circ}$. Motivated by this observation, the incidence-angle dependence of the curvature coefficients for both ion and target combinations is here fit to a heuristic functional form:

$$
\begin{gathered}
S_{X}(\theta)=A \cos (\omega \theta) \exp \left(-\frac{B}{\cos \theta}\right) . \\
S_{Y}(\theta)=A \cos (\omega \theta+C) \exp \left(-\frac{B}{\cos \theta}\right) .
\end{gathered}
$$

The coefficients $A, B, C$, and $\omega$ are fitting parameters that are allowed to vary separately with each fit. The parameter $C$ was added to Eq. (8) to address the observation that the maximum of the $S_{Y}^{\mathrm{Ge}}(\theta)$ data occurs at $20^{\circ}$ instead of $0^{\circ}$, which is unique to that data set. For $\mathrm{Si}$, however, the $\mathrm{CV}$-motivated part of the functional form, $\cos (\omega \theta+C)$, was found to have no effect on the fit of $S_{Y}^{\mathrm{Si}}(\theta)$. Thus, $\omega$ and $C$ were set to zero while fitting the $S_{Y}^{\mathrm{Si}}(\theta)$ data. The resulting fits are shown in Figs. 1 and 2. The figures also contain the results of Eqs. (5) and (6), showing the calculated $S_{X}(\theta)$ (Fig. 1) and $S_{Y}(\theta)$ (Fig. 2) curves compared to the measured values. The values calculated in the fits are given in Table I.

The figures show, for both $\mathrm{Ar}^{+} / \mathrm{Si}$ and $\mathrm{Kr}^{+} / \mathrm{Ge}$, a qualitative consistency between the measured curvature coefficients and those calculated from the opposite coefficient for bombardment angles below about $45^{\circ}$. At higher angles, the measured and calculated $S_{X}(\theta)$ curves are quite different. The $S_{X}(\theta)$ curve calculated from the curve-fitting experimental $S_{Y}$ data exhibits much larger negative values (corresponding to greater predicted instability) than do the experimental $S_{X}(\theta)$ data. This is consistent with the conclusion drawn from examining the integral relationship above - the experimental $S_{X}(\theta)$ data are not as strongly negative as they would need to be in order to satisfy the consistency requirements of the existing crater function theory. Furthermore, it is clear that the critical angles of transition from stability to instability for $1 \mathrm{keV} \mathrm{Ar}^{+} / \mathrm{Ge}$ [13], $\mathrm{Kr}^{+} / \mathrm{Ge}[14,11]$, and $\mathrm{Kr}^{+} / \mathrm{Si}[15]$ are all well above $45^{\circ}$. These high transition angles suggest that there is indeed a surfeit of stability relative to instability compared to the prediction of crater function theory.

In contrast to the predicted behavior of $S_{X}(\theta)$, the measured and calculated $S_{Y}(\theta)$ curves look reasonably similar even at high angles. We can understand this result from Eq. (5). As discussed above, the experimental $S_{X}(\theta)$ values clearly do not integrate to zero over the interval $\left[0^{\circ}-90^{\circ}\right]$ as predicted by the crater function theory. Nonetheless, the impact of this behavior on the calculation of $S_{Y}(\theta)$ in Eq. (5) is modest because the $\cot (\theta)$ term forces the calculated $S_{Y}(\theta)$ to approach zero as $\theta \rightarrow 90^{\circ}$, even if the integral does not. Thus the qualitative behavior of the calculated $S_{Y}(\theta)$ curve is not strongly sensitive to the high-angle behavior of $S_{X}(\theta)$.

In summary, experimental results for $1 \mathrm{keV} \mathrm{Ar}^{+} / \mathrm{Si}$ and $\mathrm{Kr}^{+} / \mathrm{Ge}$ appear to show qualitative agreement with the predictions of the truncated crater function theory of Ref. [6] for bombardment angles less than approximately $45^{\circ}$. For angles well above this, the instability to parallel-mode ripple formation is far weaker than would be necessary to satisfy the predictions of the crater function approach for selfconsistency, for any conceivable crater function. It is unclear at present whether this discrepancy points to a fundamental problem with the assumptions (e.g., neglect of long-range effects such as stress generated by bombardment) that enter all local response theories or is instead a consequence of the simplifications made in assuming that only $M^{(1)}(\theta)$ contributes

TABLE I. Coefficient values, with $95 \%$ confidence bounds, calculated for the data sets used in this study using the fitting functions described in Eqs. (7) and (8).

\begin{tabular}{lcccr}
\hline \hline & Fit of $S_{X, \mathrm{Si}}$ & Fit of $S_{Y, \mathrm{Si}}$ & Fit of $S_{X, \mathrm{Ge}}$ & Fit of $S_{Y, \mathrm{Ge}}$ \\
\hline$A$ & $25.43 \pm 5.13$ & $86.88 \pm 17.51$ & $58.67 \pm 18.45$ & $769.7 \pm 29.6$ \\
$B$ & $0.7535 \pm 0.181$ & $1.973 \pm 0.181$ & $0.3827 \pm 0.2763$ & $1.934 \pm 0.316$ \\
$C$ & 0 & 0 & 0 & $1.208 \pm 0.109$ \\
$\omega$ & $1.947 \pm 0.05$ & 0 & $1.441 \pm 0.069$ & $-0.253 \pm 0.159$ \\
\hline \hline
\end{tabular}


to the overall surface evolution. In either case, the use of MD simulations of individual ion impacts to predict overall surface evolution during ion bombardment will be more complicated than had been assumed - even in the linear stability limit of infinitesimal perturbations from a smooth, flat surface.

We thank Charbel S. Madi for helpful discussions. This work was performed in part at the Center for Nanoscale Systems (CNS), a member of the National Nanotechnology
Infrastructure Network (NNIN), which is supported by the National Science Foundation under NSF Award No. ECS0335765. CNS is part of Harvard University. E.A. and K.F.L. were supported by NSF DMR-1307979; J.C.P. and M.J.A. were supported by DE-FG-02-06ER46335. Use of the National Synchrotron Light Source, Brookhaven National Laboratory, was supported by the U.S. Department of Energy, Office of Science, Office of Basic Energy Sciences, under Contract No. DE-AC02-98CH10886.
[1] W. L. Chan and E. Chason, J. Appl. Phys. 101, 121301 (2007).

[2] R. M. Bradley and J. M. E. Harper, J. Vac. Sci. Technol. A 6, 2390 (1988).

[3] G. Carter and V. Vishnyakov, Phys. Rev. B 54, 17647 (1996).

[4] B. Davidovitch, M. J. Aziz, and M. P. Brenner, Phys. Rev. B 76, 205420 (2007).

[5] S. A. Norris, M. P. Brenner, and M. J. Aziz, J. Phys.: Condens. Matter 21, 224017 (2009).

[6] S. A. Norris, J. Samela, L. Bukonte, M. Backman, F. Djurabekova, K. Nordlund, C. S. Madi, M. P. Brenner, and M. J. Aziz, Nat. Commun. 2, 276 (2011).

[7] N. Kalyanasundaram, M. Ghazisaeidi, J. B. Freund, and H. T. Johnson, Appl. Phys. Lett. 92, 131909 (2008).

[8] M. Z. Hossain, K. Das, J. B. Freund, and H. T. Johnson, Appl. Phys. Lett. 99, 151913 (2011).

[9] C. S. Madi, E. Anzenberg, K. F. Ludwig, and M. J. Aziz, Phys. Rev. Lett. 106, 066101 (2011).
[10] E. Anzenberg, C. S. Madi, M. J. Aziz, and K. F. Ludwig, Jr., Phys. Rev. B 84, 214108 (2011).

[11] E. Anzenberg, J. C. Perkinson, C. S. Madi, M. J. Aziz, and K. F. Ludwig, Phys. Rev. B 86, 245412 (2012).

[12] Y. Yamamura, Y. Itikawa, and N. Itoh, Report No. IPPJ-AM-26, 1983; Y. Yamamura, C. Mössner, and H. Oechsner, Radiat. Eff. 103, 25 (1987); W. Eckstein, in Sputtering by Particle Bombardment: Experiments and Computer Calculations from Threshold to MeV Energies, edited by R. Behrisch and W. Eckstein (Springer, Berlin, 2007).

[13] M. Teichmann, J. Lorbeer, B. Ziberi, F. Frost, and B. Rauschenbach, New J. Phys. 15, 103029 (2013).

[14] J. C. Perkinson, C. S. Madi, and M. J. Aziz, J. Vac. Sci. Technol. A 31, 021405 (2013).

[15] J. C. Perkinson, E. Anzenberg, A. DeMasi, K. F. Ludwig, and M. J. Aziz (unpublished). 